\title{
A boundary value problem for implicit vector differential inclusions without assumptions of lower semicontinuity
}

Paolo Cubiotti ${ }^{1}$ and Jen-Chih $\mathrm{YaO}^{2,3^{*}}$

"Correspondence:
yaojc@mail.cmu.edu.tw
2Center for General Education,
China Medical University, Taichung,
40402, Taiwan, ROC
${ }^{3}$ Department of Mathematics, King
Abdulaziz University, P.O. Box 80203,
Jeddah, 21589, Saudi Arabia
Full list of author information is
available at the end of the article

"Correspondence:

${ }^{2}$ Center for General Education

China Medical University, Taichung, 40402, Taiwan, ROC

Abdulaziz University, PO Box 80203,

Full list of author information is

available at the end of the article

\begin{abstract}
Given a multifunction $F:[a, b] \times \mathbf{R}^{n} \times \mathbf{R}^{n} \rightarrow 2^{\mathbf{R}}$ and a function $h: X \rightarrow \mathbf{R}$ (with $X \subseteq \mathbf{R}^{n}$ ), we consider the following implicit two-point problem: find

$u \in W^{2, p}\left([a, b], \mathbf{R}^{n}\right)$ such that $\left\{\begin{array}{l}h\left(u^{\prime \prime}(t)\right) \in F\left(t, u(t), u^{\prime}(t)\right) \\ u(a)=u(b)=0\end{array}\right.$ a.e. in $[a, b]$, We prove an existence theorem where, for each $t \in[a, b]$, the multifunction $F(t, \cdot, \cdot)$ can fail to be lower semicontinuous even at all points $(x, y) \in \mathbf{R}^{n} \times \mathbf{R}^{n}$. The function $h$ is assumed to be continuous and locally nonconstant.
\end{abstract}

MSC: 34A60; 54C65; 28B20

Keywords: differential inclusions; inductively open functions; discontinuous selections; lower semicontinuity; null-measure projections

\section{Introduction}

Let $[a, b]$ be a compact interval. Given a multifunction $F:[a, b] \times \mathbf{R}^{n} \times \mathbf{R}^{n} \rightarrow 2^{\mathbf{R}}$ and a function $h: X \rightarrow \mathbf{R}$ (with $X \subseteq \mathbf{R}^{n}$ ), we are interested in the following implicit two-point problem: find $u \in W^{2, p}\left([a, b], \mathbf{R}^{n}\right)$ such that

$$
\left\{\begin{array}{l}
h\left(u^{\prime \prime}(t)\right) \in F\left(t, u(t), u^{\prime}(t)\right) \text { a.e. in }[a, b] \\
u(a)=u(b)=0_{\mathbf{R}^{n}} .
\end{array}\right.
$$

As usual, $W^{2, p}\left([a, b], \mathbf{R}^{n}\right)$ denotes the space of all functions $u \in C^{1}\left([a, b], \mathbf{R}^{n}\right)$ such that $u^{\prime}$ is absolutely continuous in $[a, b]$ and $u^{\prime \prime} \in L^{p}\left([a, b], \mathbf{R}^{n}\right)$.

Very recently, in [1], the explicit form of problem (1) has been considered, and some new results have been proved for both the multivalued problem

$$
\left\{\begin{array}{l}
u^{\prime \prime}(t) \in F\left(t, u(t), u^{\prime}(t)\right) \quad \text { a.e. in }[a, b] \\
u(a)=u(b)=0_{\mathbf{R}^{n}}
\end{array}\right.
$$

and the single-valued problem

$$
\left\{\begin{array}{l}
u^{\prime \prime}(t)=f\left(t, u(t), u^{\prime}(t)\right) \quad \text { a.e. in }[a, b] \\
u(a)=u(b)=0_{\mathbf{R}^{n}}
\end{array}\right.
$$

(c) 2015 Cubiotti and Yao. This article is distributed under the terms of the Creative Commons Attribution 4.0 International License (http://creativecommons.org/licenses/by/4.0/), which permits unrestricted use, distribution, and reproduction in any medium, provided you give appropriate credit to the original author(s) and the source, provide a link to the Creative Commons license, and indicate if changes were made. 
where $F:[a, b] \times \mathbf{R}^{n} \times \mathbf{R}^{n} \rightarrow 2^{\mathbf{R}^{n}}$ is a multifunction and $f:[a, b] \times \mathbf{R}^{n} \times \mathbf{R}^{n} \rightarrow \mathbf{R}^{n}$ is a given single-valued map.

The main peculiarity of the results proved in [1] resides in the light regularity which is required for $F$ and $f$. In particular, if the multifunction $F$ satisfies the assumptions of Theorem 1.3 of [1], then for all $t \in[a, b]$ the multifunction $F(t, \cdot, \cdot)$ can fail to be lower semicontinuous even at all points $(x, y) \in \mathbf{R}^{n} \times \mathbf{R}^{n}$. Similarly, if a function $f$ satisfies the assumptions of Theorem 2.1 of [1], then for all $t \in[a, b]$ the function $f(t, \cdot, \cdot)$ can be discontinuous even at all points $(x, y) \in \mathbf{R}^{n} \times \mathbf{R}^{n}$. Moreover, the single-valued case allows to impose less stringent requirements of measurability on $f$. For a more detailed discussion and examples, as well as for comparison with the existing literature, we refer to [1] and to the references therein.

The aim of this short note is simply to show how the results of [1], together with a deep result of B Ricceri on inductively open functions, can be put together in order to solve the implicit vector problem (1). More specifically, our aim is to prove the following existence theorem, which is in the same spirit of the results of [1] (in the sequel, if $n \in \mathbf{N}$ and $i \in$ $\{1, \ldots, 2 n\}, P_{i}: \mathbf{R}^{2 n} \rightarrow \mathbf{R}$ will denote the projection from $\mathbf{R}^{2 n}$ over the $i$ th axis; moreover, we shall denote by $\mathcal{B}\left(\mathbf{R}^{n}\right), \mathcal{L}([a, b])$ and $m_{1}$ the Borel family of $\mathbf{R}^{n}$, the family of all Lebesgue measurable subsets of $[a, b]$, and the 1-dimensional Lebesgue measure on the real line, respectively).

Theorem 1.1 Let $X \subseteq \mathbf{R}^{n}$ be a nonempty, closed, connected, and locally connected subset of $\mathbf{R}^{n}$, and let $h: X \rightarrow \mathbf{R}$ be a continuous function.

Let $F:[a, b] \times \mathbf{R}^{n} \times \mathbf{R}^{n} \rightarrow 2^{\mathbf{R}}$ be a given multifunction, $p \in\left[1,+\infty\left[, \alpha:[a, b] \rightarrow \mathbf{R}^{n}\right.\right.$ and $\beta \in L^{p}\left([a, b], \mathbf{R}^{n}\right)$ two given functions. Assume that there exist a multifunction $G:[a, b] \times$ $\mathbf{R}^{n} \times \mathbf{R}^{n} \rightarrow 2^{\mathbf{R}}$ and sets $E_{1}, \ldots, E_{2 n} \subseteq \mathbf{R}^{2 n}$, with $m_{1}\left(P_{i}\left(E_{i}\right)\right)=0$ for all $i=1, \ldots, 2 n$, such that:

(i) $G$ is $\mathcal{L}([a, b]) \otimes \mathcal{B}\left(\mathbf{R}^{n}\right) \otimes \mathcal{B}\left(\mathbf{R}^{n}\right)$-measurable with nonempty closed values;

(ii) for a.e. $t \in[a, b]$, one has

$$
\begin{gathered}
\left\{(x, y) \in \mathbf{R}^{n} \times \mathbf{R}^{n}: G(t, \cdot, \cdot) \text { is not lower semicontinuous at }(x, y)\right\} \\
\cup\left\{(x, y) \in \mathbf{R}^{n} \times \mathbf{R}^{n}: G(t, x, y) \nsubseteq F(t, x, y)\right\} \subseteq \bigcup_{i=1}^{2 n} E_{i} ;
\end{gathered}
$$

(iii) $\operatorname{int}_{X}\left(h^{-1}(t)\right)=\emptyset$, for all $t \in \operatorname{int}_{\mathbf{R}}(h(X))$;

(iv) for a.e. $t \in[a, b]$ and for all $(x, y) \in \mathbf{R}^{n} \times \mathbf{R}^{n}$, one has

$$
G(t, x, y) \subseteq h(X), \quad 0<\alpha_{i}(t) \leq \beta_{i}(t) \quad \text { for all } i=1, \ldots, n
$$

and

$$
h^{-1}(G(t, x, y)) \subseteq \prod_{i=1}^{n}\left[\alpha_{i}(t), \beta_{i}(t)\right]
$$

(where $\alpha_{i}(t)$ and $\beta_{i}(t)$ denote the ith components of the vectors $\alpha(t)$ and $\beta(t)$, respectively). 
Then there exists $u \in W^{2, p}\left([a, b], \mathbf{R}^{n}\right)$ such that

$$
\left\{\begin{array}{l}
h\left(u^{\prime \prime}(t)\right) \in F\left(t, u(t), u^{\prime}(t)\right) \quad \text { for a.e. } t \in[a, b] \\
u(a)=u(b)=0_{\mathbf{R}^{n}} .
\end{array}\right.
$$

It is immediate to check, by very simple examples, that if a multifunction $F$ satisfies the assumptions of Theorem 1.1, then for all $t \in[a, b]$ the multifunction $F(t, \cdot, \cdot)$ can fail to be lower semicontinuous even at all points $(x, y) \in \mathbf{R}^{n} \times \mathbf{R}^{n}$. In particular, when $F$ is single valued, the function $F(t, \cdot, \cdot)$ can be discontinuous at all points $(x, y) \in \mathbf{R}^{n} \times \mathbf{R}^{n}$. In the next section, two simple examples are provided in order to illustrate these circumstances. In this connection, Theorem 1.1 can be compared with Theorem 2.2 of [2] (and with the references therein), where the same problem is studied (in the single-valued case, and under the same assumptions on $h$ ) by assuming the continuity of $F$.

For the definitions and basic facts as regards multifunctions, the reader is referred to [3].

\section{The proof of Theorem 1.1}

Without loss of generality we can assume that assumptions (ii) and (iv) are satisfied for all $t \in[a, b]$. Moreover, let

$$
E:=\bigcup_{i=1}^{2 n} E_{i}
$$

Now, observe that by assumption (iii) and Theorem 2.4 of [4] the function $h$ is inductively open. That is, there exists a set $Y \subseteq X$ such that the function

$$
\left.h\right|_{Y}: Y \rightarrow h(X)
$$

is open and $h(Y)=h(X)$. It follows that the multifunction $T: h(X) \rightarrow 2^{Y}$ defined by putting, for each $s \in h(X)$,

$$
T(s)=h^{-1}(s) \cap Y
$$

is lower semicontinuous in $h(X)$ with nonempty values. To see this, fix any set $\Omega_{1} \subseteq Y$, with $\Omega_{1}$ open in the relative topology of $Y$. We get

$$
\begin{aligned}
T^{-}\left(\Omega_{1}\right) & :=\left\{s \in h(X): T(s) \cap \Omega_{1} \neq \emptyset\right\} \\
& =\left\{s \in h(X): h^{-1}(s) \cap Y \cap \Omega_{1} \neq \emptyset\right\} \\
& =\left\{s \in h(X): h^{-1}(s) \cap \Omega_{1} \neq \emptyset\right\} \\
& =h\left(\Omega_{1}\right) .
\end{aligned}
$$

Since the function $\left.h\right|_{Y}: Y \rightarrow h(X)$ is open, the set $h\left(\Omega_{1}\right)$ is open in $h(X)$. It follows that the set $T^{-}\left(\Omega_{1}\right)$ is open in $h(X)$, hence $T$ is lower semicontinuous in $h(X)$, as claimed.

Let $\Psi:[a, b] \times \mathbf{R}^{n} \times \mathbf{R}^{n} \rightarrow 2^{Y}$ be defined by

$$
\Psi(t, x, y):=T(G(t, x, y))=h^{-1}(G(t, x, y)) \cap Y
$$


(note that $\Psi$ is well defined since $G(t, x, y) \subseteq h(X)$ for all $\left.(t, x, y) \in[a, b] \times \mathbf{R}^{n} \times \mathbf{R}^{n}\right)$. We observe the following facts:

(a) the multifunction $\Psi$ is $\mathcal{L}([a, b]) \otimes \mathcal{B}\left(\mathbf{R}^{n}\right) \otimes \mathcal{B}\left(\mathbf{R}^{n}\right)$-weakly measurable; that is, for each set $\Omega \subseteq Y$, with $\Omega$ open in the relative topology of $Y$, the set

$$
\Psi^{-}(\Omega):=\left\{(t, x, y) \in[a, b] \times \mathbf{R}^{n} \times \mathbf{R}^{n}: \Psi(t, x) \cap \Omega \neq \emptyset\right\}
$$

belongs to $\mathcal{L}([a, b]) \otimes \mathcal{B}\left(\mathbf{R}^{n}\right) \otimes \mathcal{B}\left(\mathbf{R}^{n}\right)$ (this follows from Proposition 13.2.1 of [3], since $G$ is $\mathcal{L}([a, b]) \otimes \mathcal{B}\left(\mathbf{R}^{n}\right) \otimes \mathcal{B}\left(\mathbf{R}^{n}\right)$-measurable and $T$ is lower semicontinuous);

(b) $\Psi$ has nonempty values and for all $t \in[a, b]$ one has

$$
\left\{(x, y) \in \mathbf{R}^{n} \times \mathbf{R}^{n}: \Psi(t, \cdot, \cdot) \text { is not lower semicontinuous at }(x, y)\right\} \subseteq E \text {. }
$$

Indeed, if $t \in[a, b]$ and $(\bar{x}, \bar{y}) \in\left(\mathbf{R}^{n} \times \mathbf{R}^{n}\right) \backslash E$ are fixed, then the multifunction $G(t, \cdot, \cdot)$ is lower semicontinuous at $(\bar{x}, \bar{y})$, hence (by the lower semicontinuity of $T$ ) the multifunction

$$
(x, y) \in \mathbf{R}^{n} \times \mathbf{R}^{n} \rightarrow \Psi(t, x, y)=T(G(t, x, y))
$$

is lower semicontinuous at $(\bar{x}, \bar{y})$, as claimed.

Now, let $\bar{\Psi}:[a, b] \times \mathbf{R}^{n} \times \mathbf{R}^{n} \rightarrow 2^{\mathbf{R}^{n}}$ be defined by

$$
\bar{\Psi}(t, x, y):=\overline{\Psi(t, x, y)}
$$

It follows by assumption (iv) and by construction that

$$
\bar{\Psi}(t, x, y) \subseteq \prod_{i=1}^{n}\left[\alpha_{i}(t), \beta_{i}(t)\right] \quad \text { for all }(t, x, y) \in[a, b] \times \mathbf{R}^{n} \times \mathbf{R}^{n}
$$

By Proposition 2.6 and Theorem 3.5 of [5], the multifunction $\bar{\Psi}$ is $\mathcal{L}([a, b]) \otimes \mathcal{B}\left(\mathbf{R}^{n}\right) \otimes$ $\mathcal{B}\left(\mathbf{R}^{n}\right)$-measurable with nonempty values. Moreover, for all $t \in[a, b]$ one has

$$
\left\{(x, y) \in \mathbf{R}^{n} \times \mathbf{R}^{n}: \bar{\Psi}(t, \cdot, \cdot) \text { is not l.s.c. at }(x, y)\right\} \subseteq E .
$$

At this point, let us apply Theorem 2.2 of [1] to the multifunction $\bar{\Psi}$, choosing $k=2 n, T=$ $[a, b], X_{i}=\mathbf{R}$ for all $i=1, \ldots, 2 n, S=\mathbf{R}^{n}$ (all the spaces are considered with their Euclidean distance and with the usual Lebesgue measure over their Borel family), and, as above,

$$
E:=\bigcup_{i=1}^{2 n} E_{i} \subseteq \mathbf{R}^{2 n}
$$

We find that there exist $Q_{1}, \ldots, Q_{2 n} \subseteq \mathbf{R}$, with $Q_{i} \in \mathcal{B}(\mathbf{R})$ and $m_{1}\left(Q_{i}\right)=0$ for all $i=1, \ldots, 2 n$, and a function $\phi:[a, b] \times \mathbf{R}^{n} \times \mathbf{R}^{n} \rightarrow \mathbf{R}^{n}$, such that

(i) $)^{\prime} \quad \phi(t, x, y) \in \bar{\Psi}(t, x, y)$ for all $(t, x, y) \in[a, b] \times \mathbf{R}^{n} \times \mathbf{R}^{n}$;

(ii) $^{\prime}$ for all $(x, y) \in\left(\mathbf{R}^{n} \times \mathbf{R}^{n}\right) \backslash\left[\bigcup_{i=1}^{2 n}\left(P_{i}^{-1}\left(Q_{i}\right) \cup E_{i}\right)\right]$, the function $\phi(\cdot, x, y)$ is $\mathcal{L}([a, b])$ measurable; 
(iii) $^{\prime}$ for a.e. $t \in[a, b]$, one has

$$
\left\{(x, y) \in \mathbf{R}^{n} \times \mathbf{R}^{n}: \phi(t, \cdot, \cdot) \text { is discontinuous at }(x, y)\right\} \subseteq \bigcup_{i=1}^{2 n}\left(E_{i} \cup P_{i}^{-1}\left(Q_{i}\right)\right)
$$

Of course, for all $i=1, \ldots, 2 n$, one has $m_{1}\left(P_{i}\left(E_{i} \cup P_{i}^{-1}\left(Q_{i}\right)\right)\right)=0$.

Now, let us apply Theorem 2.1 of [1] with $f=g=\phi$, taking into account that for a.e. $t \in[a, b]$ and for all $(x, y) \in \mathbf{R}^{n} \times \mathbf{R}^{n}$ one has

$$
\phi(t, x, y) \in \bar{\Psi}(t, x, y) \subseteq \prod_{i=1}^{n}\left[\alpha_{i}(t), \beta_{i}(t)\right]
$$

We find that there exist $u \in W^{2, p}\left([a, b], \mathbf{R}^{n}\right)$ and a set $U_{0} \in \mathcal{L}([a, b])$, with $m_{1}\left(U_{0}\right)=0$, such that

$$
\left\{\begin{array}{l}
u^{\prime \prime}(t)=\phi\left(t, u(t), u^{\prime}(t)\right) \quad \text { for all } t \in[a, b] \backslash U_{0}, \\
u(a)=u(b)=0_{\mathbf{R}^{n}},
\end{array}\right.
$$

and also

$$
\left(u(t), u^{\prime}(t)\right) \notin \bigcup_{i=1}^{2 n}\left(E_{i} \cup P_{i}^{-1}\left(Q_{i}\right)\right) \quad \text { for all } t \in[a, b] \backslash U_{0} .
$$

In particular, by assumption (ii) we get

$$
G\left(t, u(t), u^{\prime}(t)\right) \subseteq F\left(t, u(t), u^{\prime}(t)\right) \quad \text { for all } t \in[a, b] \backslash U_{0}
$$

Consequently, taking into account the continuity of $h$ and the closedness of $X$, for all $t \in$ $[a, b] \backslash U_{0}$ we get

$$
u^{\prime \prime}(t)=\phi\left(t, u(t), u^{\prime}(t)\right) \in \bar{\Psi}\left(t, u(t), u^{\prime}(t)\right) \subseteq h^{-1}\left(G\left(t, u(t), u^{\prime}(t)\right)\right)
$$

In particular, we get

$$
h\left(u^{\prime \prime}(t)\right) \in G\left(t, u(t), u^{\prime}(t)\right) \subseteq F\left(t, u(t), u^{\prime}(t)\right)
$$

for all $t \in[a, b] \backslash U_{0}$ and hence the function $u$ satisfies our conclusion. The proof is now complete.

Remark We now give two simple examples of application of Theorem 1.1. The first example concerns with the scalar single-valued case, while the second one deals with the vector multivalued case.

Example 2.1 Let $n=1$ and let $[a, b]$ be any compact interval. Let us consider the problem

$$
\left\{\begin{array}{l}
\cos \left(u^{\prime \prime}(t)\right)=F\left(t, u(t), u^{\prime}(t)\right) \quad \text { for a.e. } t \in[a, b] \\
u(a)=u(b)=0
\end{array}\right.
$$


where $F:[a, b] \times \mathbf{R} \times \mathbf{R} \rightarrow \mathbf{R}$ is the (single-valued) function defined by putting, for each $(x, y) \in \mathbf{R}^{2}$,

$$
F(t, x, y)= \begin{cases}0 & \text { if } x \in \mathbf{Q} \text { or } y \in \mathbf{Q} \\ 1 & \text { otherwise }\end{cases}
$$

Of course, such a function $F$ (which does not depend on $t$ explicitly) is discontinuous at all points $(x, y) \in \mathbf{R}^{2}$. We observe that Theorem 1.1 can easily be applied by choosing, for any $p \in[1,+\infty[$,

$$
G:[a, b] \times \mathbf{R} \times \mathbf{R} \rightarrow \mathbf{R}, \quad G(t, x, y) \equiv 1,
$$

$E_{1}=\mathbf{Q} \times \mathbf{R}, E_{2}=\mathbf{R} \times \mathbf{Q}, X=[2 \pi, 4 \pi], \alpha(t) \equiv 2 \pi, \beta(t) \equiv 4 \pi, h(t)=\cos (t)$ (restricted to the interval $[2 \pi, 4 \pi])$. Indeed, such a function $G$ is $\mathcal{L}([a, b]) \otimes \mathcal{B}(\mathbf{R}) \otimes \mathcal{B}(\mathbf{R})$-measurable, $P_{1}\left(E_{1}\right)=P_{2}\left(E_{2}\right)=\mathbf{Q}$, and for all $t \in[a, b]$ one has

$$
\begin{gathered}
\left\{(x, y) \in \mathbf{R}^{2}: G(t, \cdot, \cdot) \text { is discontinuous at }(x, y)\right\} \\
\cup\left\{(x, y) \in \mathbf{R}^{2}: G(t, x, y) \neq F(t, x, y)\right\}=E_{1} \cup E_{2} .
\end{gathered}
$$

Moreover, for all $(t, x, y) \in[a, b] \times \mathbf{R} \times \mathbf{R}$ we have

$$
G(t, x, y)=1 \in h(X) \quad \text { and } \quad h^{-1}(G(t, x, y))=h^{-1}(1) \subseteq[2 \pi, 4 \pi]=[\alpha(t), \beta(t)] .
$$

Finally, observe that assumption (iii) is satisfied since for all $\left.t \in \operatorname{int}_{\mathbf{R}}(h(X))=\right]-1,1[$ the set $h^{-1}(t)$ contains only two points.

Consequently, by Theorem 1.1, problem (5) has a solution in $u \in W^{2, p}([a, b], \mathbf{R})$. Such a solution $u$ also satisfies the condition $u^{\prime \prime}(t) \in[2 \pi, 4 \pi]$ for a.e. $t \in[a, b]$, hence we get $u \in W^{2, \infty}([a, b], \mathbf{R})$.

Moreover, observe that Theorem 1.1 can be applied in analogous way also by taking $X=[2 \pi+4 k \pi, 4 \pi+4 k \pi]$ and $\alpha(t) \equiv 2 \pi+4 k \pi, \beta(t) \equiv 4 \pi+4 k \pi$, with $k \in \mathbf{N}$. Then, for each $k \in \mathbf{N}$, we get the existence of a solution $u_{k} \in W^{2, \infty}([a, b], \mathbf{R})$ such that $u_{k}^{\prime \prime}(t) \in[2 \pi+$ $4 k \pi, 4 \pi+4 k \pi]$ for a.e. $t \in[a, b]$. Hence, problem (5) has infinitely many solutions. Finally, we note that problem (5) does not admit the trivial solution $u(t) \equiv 0$, since $F(t, x, 0)=0$ for all $(t, x) \in[a, b] \times \mathbf{R}$ and $\cos (0)=1$.

Example 2.2 Let $n=2$, and let $\psi: \mathbf{R}^{2} \rightarrow \mathbf{R}$ be defined by $\psi(v, z)=v+z$. In what follows, we denote vectors of $\mathbf{R}^{2}$ by the notations $x:=\left(x_{1}, x_{2}\right)$ and $y:=\left(y_{1}, y_{2}\right)$. Let $E \subseteq \mathbf{R}^{4}$ be the set of vectors such that at least one component is rational, that is,

$$
E:=\left\{(x, y) \in \mathbf{R}^{4}: \text { at least one of } x_{1}, x_{2}, y_{1}, y_{2} \text { is rational }\right\}
$$

Let $F:[0,1] \times \mathbf{R}^{2} \times \mathbf{R}^{2} \rightarrow 2^{\mathbf{R}}$ be defined by

$$
F(t, x, y)= \begin{cases}1 & \text { if }(x, y) \in E, \\ {[2+t, 3+t]} & \text { if }(x, y) \notin E \text { and } x_{1}<1, \\ {[4+t, 5+t]} & \text { if }(x, y) \notin E \text { and } x_{1}>1 .\end{cases}
$$


Of course, for any fixed $t \in[0,1]$, the multifunction $F(t, \cdot, \cdot)$ is not lower semicontinuous at any point $(x, y) \in \mathbf{R}^{4}$. By Theorem 1.1, it is easily seen that for any fixed $p \in[1,+\infty[$ the problem

$$
\left\{\begin{array}{l}
\psi\left(u^{\prime \prime}(t)\right) \in F\left(t, u(t), u^{\prime}(t)\right) \quad \text { a.e. in }[0,1] \\
u(0)=u(1)=0_{\mathbf{R}^{2}}
\end{array}\right.
$$

has a solution $u \in W^{2, p}\left([0,1], \mathbf{R}^{2}\right)$. To this aim, choose $X=[1,5] \times[1,5], h:=\left.\psi\right|_{X}, \alpha(t) \equiv$ $(1,1), \beta(t) \equiv(5,5), G:[0,1] \times \mathbf{R}^{2} \times \mathbf{R}^{2} \rightarrow 2^{\mathbf{R}}$, with

$$
G(t, x, y)= \begin{cases}{[2+t, 3+t]} & \text { if } x_{1} \leq 1 \\ {[4+t, 5+t]} & \text { if } x_{1}>1\end{cases}
$$

and

$$
\begin{array}{ll}
E_{1}:=\mathbf{Q} \times \mathbf{R} \times \mathbf{R} \times \mathbf{R}, & E_{2}:=\mathbf{R} \times \mathbf{Q} \times \mathbf{R} \times \mathbf{R}, \\
E_{3}:=\mathbf{R} \times \mathbf{R} \times \mathbf{Q} \times \mathbf{R}, & E_{4}:=\mathbf{R} \times \mathbf{R} \times \mathbf{R} \times \mathbf{Q} .
\end{array}
$$

Of course, such a multifunction $G$ is $\mathcal{L}([0,1]) \otimes \mathcal{B}\left(\mathbf{R}^{2}\right) \otimes \mathcal{B}\left(\mathbf{R}^{2}\right)$-measurable with nonempty closed values. Moreover, if one fix any $t \in[0,1]$, then the multifunction $G(t, \cdot, \cdot)$ is lower semicontinuous at each point $(x, y) \in \mathbf{R}^{4}$, with $x_{1} \neq 1$. Consequently, for all $t \in[0,1]$ we have

$$
\begin{gathered}
\left\{(x, y) \in \mathbf{R}^{2} \times \mathbf{R}^{2}: G(t, \cdot, \cdot) \text { is not lower semicontinuous at }(x, y)\right\} \\
\cup\left\{(x, y) \in \mathbf{R}^{2} \times \mathbf{R}^{2}: G(t, x, y) \nsubseteq \nsubseteq F(t, x, y)\right\}=\bigcup_{i=1}^{4} E_{i}=E .
\end{gathered}
$$

Clearly, for all $i=1,2,3,4$, one has $P_{i}\left(E_{i}\right)=\mathbf{Q}$. Now, observe that for all $(t, x, y) \in[0,1] \times$ $\mathbf{R}^{2} \times \mathbf{R}^{2}$ we have

$$
G(t, x, y) \subseteq[2,6] \subseteq h(X)=[2,10]
$$

and

$$
h^{-1}(G(t, x, y)) \subseteq X=[1,5] \times[1,5]=\left[\alpha_{1}(t), \beta_{1}(t)\right] \times\left[\alpha_{2}(t), \beta_{2}(t)\right] .
$$

Finally, let us show that for all $\left.s \in \operatorname{int}_{\mathbf{R}}(h(X))=\right] 2,10$ [, we have $\operatorname{int}_{X}\left(h^{-1}(s)\right)=\emptyset$ (though this fact is quite intuitive - since $h$ is never locally constant - we shall provide an explicit proof). To this aim, fix $s \in \operatorname{int}_{\mathbf{R}}(h(X))$, and let $\left(v_{0}, z_{0}\right) \in h^{-1}(s)$. Therefore, $\left(v_{0}, z_{0}\right) \in X$ and $h\left(v_{0}, z_{0}\right)=v_{0}+z_{0}=s$. Let $\Omega$ be an open set in $X$ such that $\left(v_{0}, z_{0}\right) \in \Omega$. Of course, one can find $r>0$ such that

$$
\left(\left[v_{0}-r, v_{0}+r\right] \times\left[z_{0}-r, z_{0}+r\right]\right) \cap X \subseteq \Omega .
$$


Let $v_{1}$ be any point in $\left[v_{0}-r, v_{0}+r\right] \cap[1,5]$, with $v_{1} \neq v_{0}$. We have

$$
\left(v_{1}, z_{0}\right) \in \Omega
$$

and

$$
h\left(v_{1}, z_{0}\right)=v_{1}+z_{0} \neq v_{0}+z_{0}=s
$$

Hence, the set $h^{-1}(s)$ has empty interior in $X$, as claimed. Thus, all the assumptions of Theorem 1.1 are satisfied. Consequently, problem (6) has at least a solution $u \in W^{2, p}\left([0,1], \mathbf{R}^{2}\right)$. As a matter of fact, since $u^{\prime \prime}(t) \in X$ for a.e. $t \in[0,1]$, we get $u \in W^{2, \infty}\left([0,1], \mathbf{R}^{2}\right)$. As before, we note that problem (6) does not admit the trivial solution $u(t) \equiv 0_{\mathbf{R}^{2}}$.

\section{Competing interests}

The authors declare that they have no competing interests.

\section{Authors' contributions}

All authors contributed equally and significantly in writing this article. All authors read and approved the final manuscript.

\section{Author details}

'Department of Mathematics and Computer Science, University of Messina, Viale F. Stagno d'Alcontres 31, Messina, 98166, Italy. ${ }^{2}$ Center for General Education, China Medical University, Taichung, 40402, Taiwan, ROC. ${ }^{3}$ Department of Mathematics, King Abdulaziz University, P.O. Box 80203, Jeddah, 21589, Saudi Arabia.

\section{Acknowledgements}

The second author was partially supported by the Grant MOST 103-2923-E-039-001-MY3.

Received: 26 February 2015 Accepted: 19 May 2015 Published online: 10 June 2015

\section{References}

1. Cubiotti, P, Yao, JC: Two-point problem for vector differential inclusions with discontinuous right-hand side. Appl. Anal. 93, 1811-1823 (2014)

2. Marano, SA: On a boundary value problem for the differential equation $f\left(x, u, u^{\prime}, u^{\prime \prime}\right)=0$. J. Math. Anal. Appl. 182, 309-319 (1994)

3. Klein, E, Thompson, AC: Theory of Correspondences. Wiley, New York (1984)

4. Ricceri, B: Sur la semi-continuité inférieure de certaines multifonctions. C. R. Acad. Sci. Paris 294, 265-267 (1982)

5. Himmelberg, CJ: Measurable relations. Fundam. Math. 87, 53-72 (1975)

\section{Submit your manuscript to a SpringerOpen ${ }^{\circ}$ journal and benefit from:}

- Convenient online submission

Rigorous peer review

- Immediate publication on acceptance

- Open access: articles freely available online

- High visibility within the field

- Retaining the copyright to your article 